\title{
Nonoverlay Heterogeneous Network Planning for Energy Efficiency
}

\author{
Mahmut Demirtaş and Alkan Soysal \\ Electrical and Electronics Engineering, Bahçeşehir University, Istanbul, Turkey \\ Correspondence should be addressed to Alkan Soysal; alkan.soysal@eng.bau.edu.tr
}

Received 27 July 2016; Revised 27 December 2016; Accepted 22 January 2017; Published 9 February 2017

Academic Editor: Simone Morosi

Copyright ( 2017 Mahmut Demirtaş and Alkan Soysal. This is an open access article distributed under the Creative Commons Attribution License, which permits unrestricted use, distribution, and reproduction in any medium, provided the original work is properly cited.

\begin{abstract}
In this paper, we introduce nonoverlay microcell/macrocell planning that is optimally designed for improving energy efficiency of the overall heterogeneous cellular network. We consider two deployment strategies. The first one is based on a fixed hexagonal grid and the second one is based on a stochastic geometry. In both of our models, microcells are placed in those areas where the received signal power levels of macrocell common pilot channels are below a certain threshold. Thus, interference between microcells and macrocells is minimized. As a result, addition of microcells increases the achieved number of bits per unit energy. Under such deployment assumptions, we investigate the effects of certain parameters on the energy efficiency. These parameters include the user traffic, the Intersite Distance (ISD), the size of microcells and the number of microcells per macrocell for the grid model, and macrocell density and microcell density for the stochastic model. The results of our performance analyses show that utilizing microcells in a sparse user scenario is worse for the energy efficiency whereas it significantly improves both energy and spectral efficiencies in a dense user scenario. Another interesting observation is that it is possible to choose an optimum number of microcells for a given macrocell density.
\end{abstract}

\section{Introduction}

For a long period of time, the main concern of cellular systems was to increase the spectral efficiency. Among several others, one way to increase the spectral efficiency is to overlay microcells on the existing macrocell coverage area. This two-tier approach guarantees the coverage and increases the spectral efficiency of the users that are close to microcell stations. However, the downside of this approach is that the energy efficiency of the network gets worse by the addition of new overlaid microcells.

Over the last couple of years, the energy efficiency of cellular networks has been an increasing concern because of its environmental and operational cost effects. In order to improve the energy efficiency, several solutions are proposed in the literature. Detailed approach to general energy efficiency problem can be found in [1-5] and the references therein.

The performance of an energy efficiency analysis depends strongly on the definition of energy efficiency metric [6].
Area power consumption and bit per joule are the two most common energy efficiency metrics that are considered in the literature. Similar to [7-10], we considered area power consumption metric in some previous studies [11, 12]. However, other studies report that bit per joule metric captures the energy efficiency in high-load conditions better than area power consumption metric [2]. In the literature, there are several works where the authors employed bit per joule as their efficiency metric [13-17]. We also use bit per joule metric in this study, since our focus is to obtain energy efficient methods for increasingly high demands of spectral efficiency.

Another important concept in the energy efficiency analysis of heterogeneous cellular networks is the base station deployment model. In the literature, generally, two different models are used to determine the locations of base stations: fixed hexagonal grid model and Poisson Point Process (PPP) based stochastic geometry model. Although stochastic geometry models are better fit to real base station deployments, fixed hexagonal grid models can provide a better insight to the mathematical problem. 
In [7-12], fixed hexagonal grid model is employed and area power consumption metric is used for energy efficiency comparison. The authors in $[7,8]$ find the optimum ISD between macrocells when several microcells are overlaid on the macrocell coverage area. However, in such a scenario, microcell addition always increases the total power consumption of the system because of the overlay structure. In [9], overlaid microcells or reduced range omnidirectional macrocells are turned on and off depending on a traffic model. The authors find the optimum ISD for a range of path loss exponent values. In [10], microcell base station planning is considered; however the optimization problem is to minimize the number of microcell base stations over a set of candidate microcell base station positions and a traffic constraint. Therefore, improving energy efficiency is not the main goal in [10]. In [11, 12], we found optimum ISD in a nonoverlay microcell deployment.

There are also some studies that consider bit per joule metric for energy efficiency analysis. In $[13,15]$, the authors consider a single cell OFDMA network and investigate the trade-off between spectral efficiency and bit per joule energy efficiency. In [16, 17], the authors extend their results to a case where there is a single macrocell base station with several uniformly overlaid small cells and to multiple input multiple output (MIMO) broadcast channels, respectively. In a multicell scenario and for a fixed grid, [14] assumes a homogeneous network deployment with microcell or picocell base stations and calculates the effect of backhaul power consumption on the bit per joule energy efficiency. In [18], we considered a heterogeneous network with a fixed hexagonal grid and showed that bit per joule energy efficiency increases with increasing number of microcells.

Stochastic geometry based models are considered in [1922]. In [19], macrocells are located according to a PPP in a Euclidean plane. The authors consider only homogeneous macrocell networks, and their goal is to find tractable coverage and rate expressions. These mathematical analyses of coverage and average rates are extended to heterogeneous networks in [20]. The model in [21] assumes a single macrocell and several small cells that are distributed according to a PPP. The authors find the optimal density of small cells that maximize energy efficiency. Similar results for multicell overlaid heterogeneous networks are derived in [22], where energy efficiency metric is area power consumption.

In this paper, we improve the energy efficiency through a nonoverlay planning of heterogeneous networks. We deploy microcells at the locations where the received signal strength is expected to be relatively low. In the fixed grid model, the microcell locations are chosen to be the cell edges of the hexagonal cell site. In the stochastic geometry model, we employ a two-stage deployment. In the first stage, macrocells are placed according to a PPP and the coverage regions are determined. In the second stage, we detect the regions where the received signal strength is lower than a certain limit. Then, we place the microcells on those regions according to a separate PPP and update the coverage regions.

Due to the nonoverlay nature of our deployment, a macrocell base station saves power when microcells are deployed in a site. Using this model, we calculate the energy efficiency as a function of ISD (or macrocell density), the number of microcells (or microcell density), and the size of microcells. For power consumption modeling, we use comprehensive power consumption models that are introduced in [23]. We consider bit per joule as our energy efficiency metric. Through our simulations, we observe that deploying microcells simultaneously increases both energy efficiency and spectral efficiency. Also, we conclude that it is possible to choose intervals for ISD (or macrocell density) and number of microcells (or microcell density) that improves the energy efficiency the most.

\section{System Model}

In this paper, our goal is to improve the energy efficiency of a nonoverlay heterogeneous cellular network without compromising the spectral efficiency. Here, "heterogeneous cellular network" refers to a single technology network that contains different sizes of base stations. We assume a fixed coverage constraint that guarantees that a certain minimum percentage of a service area is covered. In addition, we assume that all base stations work under full-load condition.

In order to investigate the heterogeneous networks in terms of energy and spectral efficiency, we use two different models for base station deployment: a fixed hexagonal grid model and a stochastic geometry based model. In the first model, we consider a hexagonal grid of macrocells where each macrocell receives interference from a tier of neighboring macrocells. Due to the nonoverlay nature of microcell deployment and in order to save power, the radius of a macrocell might get smaller as the number of microcells increases. This can be observed in Figures 1(a), 1(c), and 1(e), where the coverage constraint is $100 \%$ for all subfigures. ISD determines the hexagonal cell size, and coverage area determines the macrocell radius, $R_{m}$ (see Figure 1(a)). Microcells are deployed along the edges of the hexagons (see Figures 1(c) and 1(e)). Our goal is to analyze the energy efficiency of such a deployment over certain parameters like ISD, number of microcells, and microcell radius. We also consider different user densities in order to observe the effect of microcell utilization. In the sparse scenario, we have 5 users $/ \mathrm{km}^{2}$, whereas in the dense scenario, we have 100 users $/ \mathrm{km}^{2}$.

In the second model, we use two separate Poisson point processes for macrocells and microcells with densities of $\lambda_{M}$ and $\lambda_{\mu}$, respectively. A $10 \times 10 \mathrm{~km}^{2}$ area is chosen for the analysis. First, macrocells are located with the density of $\lambda_{M}$ and the coverage regions are determined according to the received signal strength from the common pilot channel (see Figure 1(b)). A point is said to be covered if the received signal strength from the pilot channel of the closest base station is above a certain limit, namely, $P_{c}$. The allocated power ratio for the pilot channel is $10 \%$ of the total power budget of each base station. If the pilot channel power is not enough to satisfy the coverage condition for a particular scenario, we increase the pilot signal strength by a step size of $2 \%$ of total power budget. At the second stage, the regions for which the received signal strength is lower than another certain limit, namely, $P_{\mu}$, are detected. Then, microcells are deployed on those regions, with the density of $\lambda_{\mu}$, and the coverage regions are updated 




— Hexagonal cell site — Macrocell coverage regions * Macrocell locations

(a) Grid model, homogeneous deployment



Distance $(\mathrm{m})$

- Hexagonal cell site _ Macrocell coverage regions

* Macrocell locations _ Microcell coverage regions

- Microcell locations

(c) Grid model, 2 microcells per site



Distance (m)



(e) Grid model, 8 microcells per site



* Macrocell locations

— Macrocell coverage regions

(b) PPP model, homogeneous deployment



* Macrocell locations _ Macrocell coverage regions

- Microcell locations _ Microcell coverage regions

(d) PPP model, 2 microcells per site



* Macrocell locations _ Macrocell coverage regions

- Microcell locations _ Microcell coverage regions

(f) PPP model, 8 microcells per site

FIgURE 1: Examples of macrocell and microcell deployment. 
TABLE 1: Urban macrocell and microcell path loss models.

\begin{tabular}{|c|c|c|c|c|c|}
\hline & & Path loss & & $\begin{array}{c}\text { Shadowing } \\
\sigma_{(10 \log \xi)}\end{array}$ & $\begin{array}{l}\text { Fast fading } \\
\text { margin }(\zeta)\end{array}$ \\
\hline \multirow[b]{2}{*}{$\begin{array}{l}\text { Urban } \\
\text { macrocell }\end{array}$} & LOS & $\begin{array}{c}\mathrm{PL}=22 \log (r)+28+20 \log \left(f_{c}\right) \\
\mathrm{PL}=40 \log \left(r_{1}\right)+7.8-18 \log \left(h_{\mathrm{BS}}^{\prime}\right)-18 \log \left(h_{\mathrm{UT}}^{\prime}\right)+2 \log \left(f_{c}\right)\end{array}$ & $\begin{aligned} 10<r & <r_{\mathrm{BP}}^{\prime} \\
r_{\mathrm{BP}}^{\prime}<r_{1} & <5000\end{aligned}$ & $4 \mathrm{~dB}$ & \multirow{4}{*}{$2 \mathrm{~dB}$} \\
\hline & NLOS & $\begin{array}{l}\mathrm{PL}=161.04-7.1 \log (W)+7.5 \log (h)-\left(24.37-3.7\left(\frac{h}{h_{\mathrm{BS}}}\right)^{2}\right) \log \left(h_{\mathrm{BS}}\right)+ \\
\left(43.42-3.1 \log \left(h_{\mathrm{BS}}\right)\right)(\log (r)-3)+20 \log \left(f_{c}\right)-\left(3.2 \log \left(11.75 h_{\mathrm{UT}}\right)\right)^{2}-4.97\end{array}$ & $10<r<5000$ & $6 \mathrm{~dB}$ & \\
\hline \multirow{2}{*}{$\begin{array}{l}\text { Urban } \\
\text { microcell }\end{array}$} & LOS & $\begin{array}{c}\mathrm{PL}=22 \log (r)+28+20 \log \left(f_{c}\right) \\
\mathrm{PL}=40 \log \left(r_{1}\right)+7.8-18 \log \left(h_{\mathrm{BS}}^{\prime}\right)-18 \log \left(h_{\mathrm{UT}}^{\prime}\right)+2 \log \left(f_{c}\right)\end{array}$ & $\begin{aligned} 10 & <r<r_{\mathrm{BP}}^{\prime} \\
r_{\mathrm{BP}}^{\prime} & <r_{1}<5000\end{aligned}$ & $3 \mathrm{~dB}$ & \\
\hline & NLOS & $\mathrm{PL}=36.7 \log (r)+22.7+26 \log \left(f_{c}\right)$ & $10<r<2000$ & $4 \mathrm{~dB}$ & \\
\hline
\end{tabular}

(see Figures 1(d) and 1(f)). It is important to note that $P_{\mu}$ is always larger than $P_{c}$. Thus, the regions that can be covered by macrocells shrink as it is in the grid model. By this method, we keep nonoverlaying structure as much as possible.

2.1. Power Consumption Models. For an accurate energy efficiency analysis, a power consumption model needs to include power consumed at the base stations due to signal processing, cooling, network transmission, and so forth, in addition to the Radio Frequency (RF) transmitted power. The more detailed power consumption modeling is done, the more accurate our analysis will become.

The RF transmit power is allocated mainly between the common pilot channel and the traffic channels. The common pilot channel received power determines the coverage area and traffic channel received power determines the data rate that is provided to the mobile stations. In order to calculate the received power at the mobile stations, we consider deterministic path loss, shadowing, and fast fading effects. The received signal strength (in $\mathrm{dB}$ scale) is given as a function of the distance between transmitter and receiver, $r$, as

$$
P_{\mathrm{rx}}(r)=P_{\mathrm{tx}}-\mathrm{PL}(r)-\xi-\zeta
$$

where $P_{\mathrm{tx}}$ is the transmitted signal strength, $\mathrm{PL}(r)$ is the path loss, $\xi$ is the log-normal shadowing variable, and $\zeta$ is the random fast fading variable. For our coverage calculations, we use urban macrocell and microcell path loss models, shadowing variance, fast fading margin, carrier frequency, and antenna height values that are given in [24]. In Table 1, a complete summary of our propagation model is given.

In this model, the Line of Sight (LOS) probability for a macrocell, $\operatorname{Pr}_{M}$ (LOS), is [24]

$$
\operatorname{Pr}_{M}(\mathrm{LOS})=\min \left\{\frac{18}{r}, 1\right\}\left(1-e^{-r / 63}\right)+e^{-r / 63},
$$

and the LOS probability for microcells, $\operatorname{Pr}_{\mu}$ (LOS), is [24]

$$
\operatorname{Pr}_{\mu}(\mathrm{LOS})=\min \left\{\frac{18}{r}, 1\right\}\left(1-e^{-r / 36}\right)+e^{-r / 36},
$$

where $r$ is the distance between the user and serving base station.

As stated above, RF transmit power is only a fraction of the total power consumed by a base station. In order to include the effects of baseband signal processing, transmission, cooling, and so forth, we consider the power consumption model that is introduced in [23] as follows:

$$
P_{i}=N_{\mathrm{TX}, i}\left(P_{0, i}+\Delta_{i} P_{\mathrm{tx}, i}\right), \quad 0<P_{\mathrm{tx}, i} \leq P_{\mathrm{max}, i}
$$

where $i=M$ and $i=\mu$ correspond to macrocells and microcells, respectively; $N_{\mathrm{TX}, i}$ is the number of transceiver chains, $P_{0, i}$ is the power consumption at zero RF output power, $\Delta_{i}$ is the slope of the load dependent power consumption, and $P_{\max , i}$ is the maximum power budget of base stations. Here, the constant term $P_{0, i}$ includes power consumed at the base stations due to signal processing, cooling, backhaul, and so forth.

2.2. Energy Efficiency Metric. In literature, area power consumption is frequently used as an efficiency metric for wireless cellular networks. It is defined as the ratio of the total power consumption and the total service area of a network. This metric has the advantage of capturing the size of the total service area. However, it cannot capture the effect of the total data rate that is provided in the same service area. In order to capture both effects, in this work, we consider the ratio of area spectral efficiency to area power consumption as our performance metric

$$
\begin{aligned}
\mathrm{EE} & =\frac{\text { Area Spectral Efficiency }}{\text { Area Power Consumption }}\left(\frac{\mathrm{bits} / \mathrm{s} / \mathrm{Hz} / \mathrm{km}^{2}}{\text { Watts } / \mathrm{km}^{2}}\right. \\
& =\text { bits } / \text { joule } / \mathrm{Hz})
\end{aligned}
$$

Area power consumption is the per unit area total power consumed by all base stations and is calculated by summing (4) over all macrocell and microcell base stations in a given service area. Area spectral efficiency was defined as the total data rate per unit area and per unit bandwidth that is provided by a base station in [25]. Since there are many base stations in a service area, we use the following formula [8] to evaluate the total area spectral efficiency:

$$
\mathrm{ASE}=\sum_{i} S_{i} \operatorname{Pr}\left\{N_{i}>0\right\},
$$

where the summation is over all macrocell and microcell base stations, $S_{i}$ is the area spectral efficiency of base station $i$, and 
TABLE 2: Parameters for path loss models.

\begin{tabular}{lccc}
\hline \multicolumn{1}{c}{ Urban macrocell } & & Urban microcell \\
\hline$P_{c}$ (receiver sensitivity) & $-120 \mathrm{dBm}$ & $P_{c}$ (receiver sensitivity) & $-120 \mathrm{dBm}$ \\
$f_{c}$ (carrier frequency in $\left.\mathrm{GHz}\right)$ & $2.4 \mathrm{GHz}$ & $f_{c}$ (carrier frequency in $\mathrm{GHz}$ ) & $2.4 \mathrm{GHz}$ \\
$h_{\mathrm{BS}}$ (base station antenna height) & $25 \mathrm{~m}$ & $h_{\mathrm{BS}}$ (base station antenna height) & $10 \mathrm{~m}$ \\
$h_{\mathrm{UT}}$ (user terminal antenna height) & $1.5 \mathrm{~m}$ & $h_{\mathrm{UT}}$ (user terminal antenna height) & $1.5 \mathrm{~m}$ \\
$h_{\mathrm{BS}}^{\prime}$ (effective BS antenna height) & $24 \mathrm{~m}$ & $h_{\mathrm{BS}}^{\prime}$ (effective BS antenna height) & $9 \mathrm{~m}$ \\
$h_{\mathrm{UT}}^{\prime}$ (effective UT antenna height) & $0.5 \mathrm{~m}$ & $h_{\mathrm{UT}}^{\prime}$ (effective UT antenna height) & $r_{\mathrm{BP}}^{\prime}$ (break point distance) \\
$r_{\mathrm{BP}}^{\prime}$ (break point distance) & $4 h_{\mathrm{BS}}^{\prime} h_{\mathrm{UT}}^{\prime} f_{c} / c \mathrm{~m}$ & & \\
$h$ (average building height) & $20 \mathrm{~m}$ & & \\
$W$ (street width) & $20 \mathrm{~m}$ & & \\
\hline
\end{tabular}

$N_{i}$ is the number of uniformly distributed users in the service area of base station $i$.

The area spectral efficiency of a particular base station, $S_{i}$, is the per unit area total achievable data rate that is calculated over the users in the service area of that base station, and it is given as

$$
S_{i}=\frac{1}{A_{C}} \mathbb{E}\left[\sum_{u=1}^{N_{i}} \log _{2}\left(1+\gamma_{u}\left(r_{u i}\right)\right)\right],
$$

where $\gamma_{u}\left(r_{u i}\right)$ is the Signal to Interference and Noise Ratio (SINR) of user $u, r_{u i}$ is the distance of user $u$ to base station $i$, and $A_{C}$ is the total service area.

Here, we assume a universal frequency reuse. In other words, all signals from adjacent macrocells and microcells to base station $i$ contribute to the interference level. As a result, we define the SINR level of user $u$ that is served by base station $i$ as

$$
\gamma_{u}\left(r_{u i}\right)=\frac{P_{\mathrm{rx}, u}\left(r_{u i}\right)}{\sum_{j \neq i} P_{\mathrm{rx}, u}\left(r_{u j}\right)+P_{N}},
$$

where $P_{\mathrm{rx}, u}\left(r_{u i}\right)$ is the received power level from base station $i$ to user $u$ and it can be calculated using (1).

\section{Hexagonal Grid Model}

In this section, we calculate the energy efficiency performance of hexagonal grid deployment model over downlink channels using Monte Carlo simulations. We generate a large number of uniformly distributed users in the service area. Then, we calculate energy efficiency and spectral efficiency of the network as a function of ISD, number of microcells, user density, and microcell size. We assume that the coverage constraint is $95 \%$. We consider the macrocell ISD to be from $500 \mathrm{~m}$ to $1500 \mathrm{~m}$ in order to reflect typical macrocell sizes. The parameters that we use in power consumption model are given as the following: $N_{\mathrm{TX}, M}=6, P_{0, M}=130$ Watts, $\Delta_{M}=4.7, P_{\max , M}=20$ Watts, $N_{\mathrm{TX}, \mu}=2, P_{0, \mu}=56$ Watts, $\Delta_{\mu}=2.6$, and $P_{\max , \mu}=6.3$ Watts [23]. In addition, path loss parameters are given in Table 2 [24].

In Figure 2, we plot the energy efficiency of several microcell density scenarios with respect to ISD. We assume that the size of a microcell is $20 \%$ of the size of a macrocell.
We start with the sparse scenario with user density of 5 users $/ \mathrm{km}^{2}$. First observation of Figure 2(a) is that the energy efficiency improves with increasing ISD, except for the homogeneous scenario. However, deploying microcells results in a worse energy efficiency under sparse user assumption and for low ISD values. This is basically a result of many microcells operating under no load condition but spending considerable amount of power just to stay on. For low ISD values, the power of those microcells with no users contributes negatively (in the form of interference) to the spectral efficiency of neighbor cells but does not provide spectral efficiency for its own cell. Therefore we conclude that utilizing microcells for low traffic areas is not necessary in a fixed hexagonal deployment with small ISD.

Next, we analyze the performance of a dense user scenario with 100 users $/ \mathrm{km}^{2}$. We observe in Figure 2(b) that, similar to sparse user case, the energy efficiency improves with increasing ISD. However, for a given ISD, the energy efficiency is much better in dense user case than it is in sparse user case. The reason for this is that most microcells are fully utilized under dense user scenarios. Another observation is that the energy efficiency is not monotonic in the number of microcells. After some point, adding more microcells does not further improve the energy efficiency. Therefore, there must be an optimum number of microcells as a function of user traffic in a given area. For the scenario in Figure 2(b), 8 microcells/site is almost 5 times more energy efficient than macrocell-only network.

In a dense user scenario increasing the number of nonoverlay microcells improves the energy efficiency, because macrocells shrink their coverage area in order to save some transmission power and do not spent power to service cell edge users. However, we should also investigate how much this approach affects the spectral efficiency. We first consider the sparse scenario with 5 users $/ \mathrm{km}^{2}$. In Figure 3(a), we observe that the spectral efficiency decreases with increasing ISD. The reason for this is the decrease in expected received signal strength with the increase in cell size. For the pure macrocell case, the decrease in spectral efficiency is much faster. We also observe that 8 microcells/site is the most spectral efficient scenario. In this analysis we do not include higher microcell densities than 11 microcells/site, because the microcells start to overlap considerably when we deploy more than 11 microcells per site. Next, we consider a dense 




(a) 5 users $/ \mathrm{km}^{2}$



(b) 100 users $/ \mathrm{km}^{2}$

FIGURE 2: Energy efficiency for $20 \%$ microcell size.

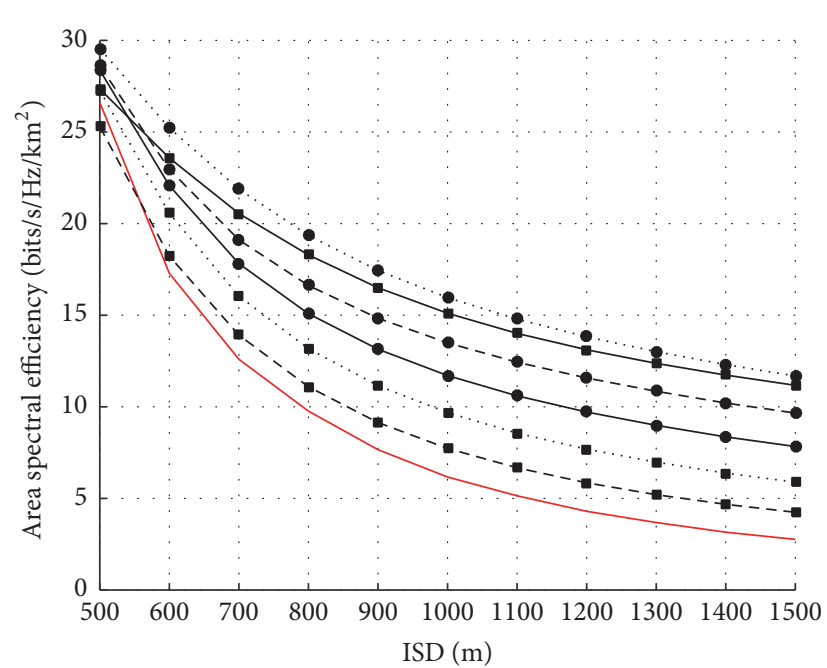



(a) 5 users $/ \mathrm{km}^{2}$


(b) $100 \mathrm{users} / \mathrm{km}^{2}$

Figure 3: Area spectral efficiency for $20 \%$ microcell size.

scenario with 100 users $/ \mathrm{km}^{2}$. In Figure 3(b), we again observe that the spectral efficiency decreases with increasing ISD. However, it is important to note that the area spectral efficiency significantly increases with the increasing user density.

Spectral efficiency results confirm that utilizing nonoverlay microcells is beneficial under dense user scenarios.
Combining this with the energy efficiency performance, we conclude that utilizing nonoverlay microcells improves both spectral efficiency and energy efficiency under high user traffic conditions. On the other hand, increasing ISD improves energy efficiency while it reduces spectral efficiency. In the remaining analysis, we fix the ISD at $500 \mathrm{~m}$ to achieve the highest possible spectral efficiency. 


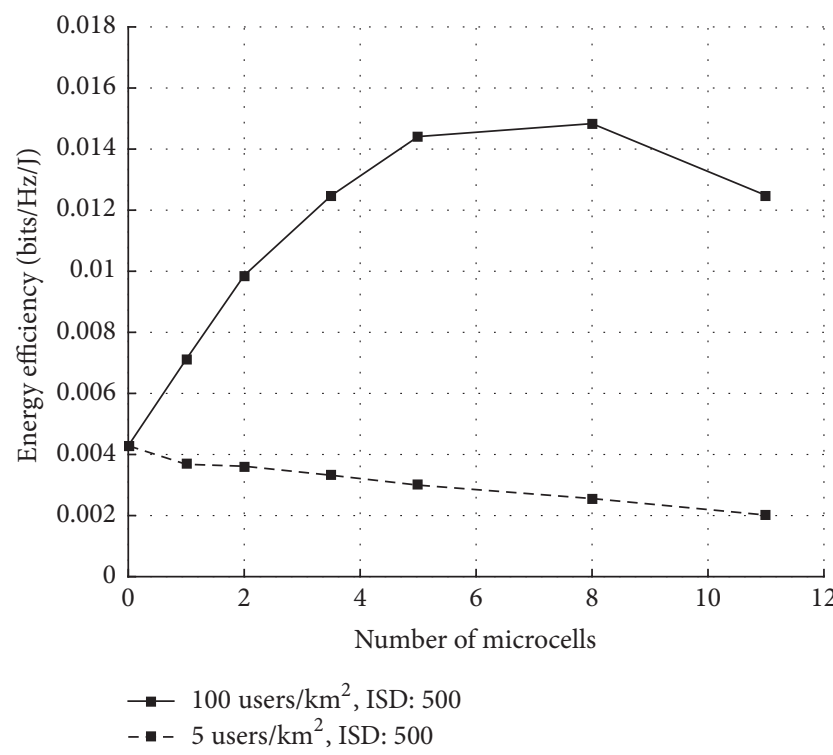

(a) Energy efficiency



(b) Area spectral efficiency

Figure 4: The effect of the number of microcells when ISD $=500 \mathrm{~m}$ and microcell size is $20 \%$.



- - 1 microcell per site

-. 2 microcells per site

$\multimap 3.5$ microcells per site

- - 5 microcells per site

- 8 microcells per site

- 11 microcells per site

(a) Energy efficiency



- - 1 microcell per site $\quad$ - - 5 microcells per site

- 2 microcells per site $\quad$-.. 8 microcells per site

$\multimap 3.5$ microcells per site $\rightarrow 11$ microcells per site

(b) Area spectral efficiency

FIGURE 5: The effect of microcells size for ISD $=500 \mathrm{~m}$ and dense user scenario.

In order to determine the optimum number of microcells per site, we plot the energy efficiency and spectral efficiency graphs with respect to the number of microcells in Figure 4. Once again we observe that addition of microcells hurts the energy efficiency for sparse user scenario. For dense user scenario, 8 microcells per macrocell result in the maximum energy efficiency (see Figure 4(a)). When we observe the spectral efficiency in Figure 4(b), we conclude that 8 microcells per macrocell is the best choice when the microcell size is $20 \%$ of the hexagon size. It is important to note that
8 microcells per site is not an arbitrary number. It is the minimum number of microcells per site that can be deployed around the hexagon site without any gaps between microcells (see Figure 1(e)).

A natural question to ask at this point is how the energy efficiency and spectral efficiency are affected by the ratio of hexagonal site size to microcell size. In a dense user scenario and for the best energy efficiency and spectral efficiency, we observe in Figure 5 that the microcell radius should be $30 \%$ of the macrocell radius when the number of microcells per site 




-. 8 microcells per site, ISD: 500

(a) Energy efficiency

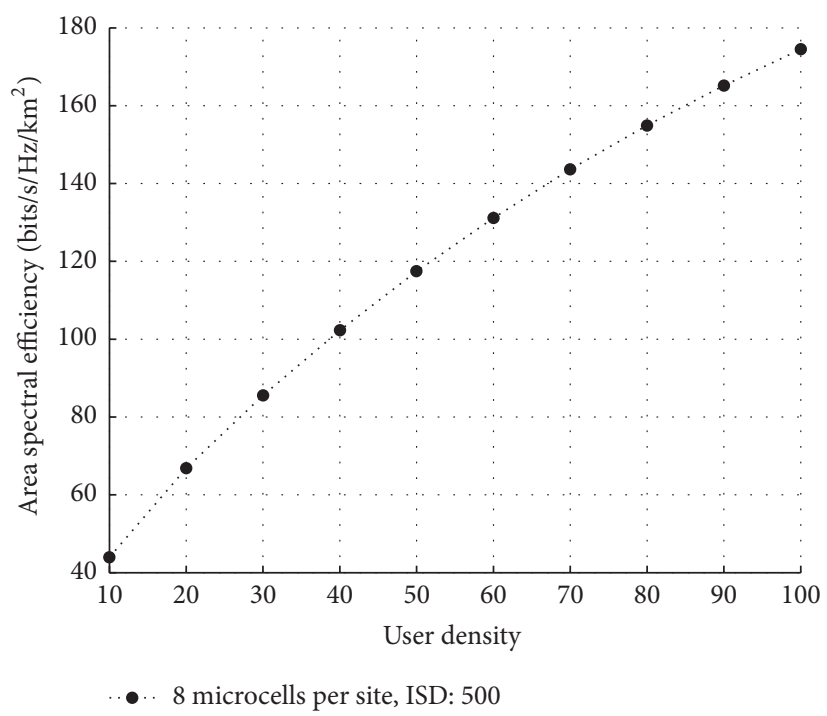

(b) Area spectral efficiency

FIGURE 6: The effect of user density for ISD $=500 \mathrm{~m}$, microcell size of $20 \%$, and the number of microcells $=8$.

is less than 5 . On the other hand, microcell radius should be $20 \%$ of the macrocell radius when the number of microcells is larger than 5. Finally, we observe that the combination of having 8 microcells per site and microcell size of $20 \%$ seems to be the best scenario in terms of both energy efficiency and spectral efficiency.

Finally, we fix the best combination that is mentioned above and in Figure 6 and plotted the energy efficiency and spectral efficiency as a function of user density. We observe that both energy efficiency and spectral efficiency are monotone increasing functions of the user density. In this fixed hexagonal structure, idle microcells are the main reason of having worse energy efficiency. As the user density increases, the probability of having idle microcells decreases.

\section{Stochastic Geometry Based Model}

In this section, we calculate the energy efficiency performance of stochastic geometry based deployment model over downlink channels using Monte Carlo simulations. As described in Section 2, locations of macrocell and microcell base stations are determined according to separate Poisson point processes with $\lambda_{M}$ and $\lambda_{\mu}$. In order to compare the results of the fixed grid and stochastic geometry based models, each $\lambda_{M}$ in the stochastic geometry model is chosen so that the number of macrocells per unit area is the same in both models. In a similar manner, each $\lambda_{\mu}$ is evaluated by multiplying $\lambda_{M}$ with the ratio of number of microcells to the number of macrocells in the grid model. Similar to the fixed grid case, we generate a large number of uniformly distributed users inside the service area. Then, we calculate energy efficiency and spectral efficiency of the network as a function of macrocell, microcell, and user densities. We assume that the coverage constraint is $95 \%$. In addition to the parameters that are given in Table 2, we use $P_{\mu}=-90 \mathrm{dBm}$.

In Figure 7, we demonstrate the energy efficiency performances of several heterogeneous scenarios with respect to macrocell density. It is important to note that macrocell density and ISD are inversely proportional. For a sparse user case, in Figure 7(a), the energy efficiency in stochastic geometry model improves with increasing average ISD (or decreasing macrocell density), similar to the fixed hexagonal grid model. However, energy efficiency of the stochastic model is considerably worse than that of the grid model. For example, in a homogeneous network, the energy efficiency of the grid model is almost double of the energy efficiency of the stochastic model. In Figure 7(b), we observe that the effect of a dense user distribution is not as drastic in stochastic model as it was in the grid model. However, deploying a convenient number of microcells results in an improved energy efficiency. Overall, we can conclude from Figure 7 that nonoverlay heterogeneous deployment has better energy efficiency in stochastic model for both sparse and dense user scenarios.

Next, similar to the grid model, we investigate the effect of our nonoverlay stochastic geometry based deployment model on the area spectral efficiency. Figures 8(a) and 8(b) show that increasing both macrocell and microcell densities improves the area spectral efficiency, for both sparse and dense user scenarios, respectively. In Figure 8(a), 8 microcells/site provides 2 times better area spectral efficiency than the homogeneous scenario for $\lambda_{M}=1.1547$. In Figure 8(b), 11 microcells/site provides 3 times better area spectral efficiency than the homogeneous scenario for $\lambda_{M}=1.1547$. Thus, $\lambda_{M}$ is fixed at 1,1547 in the rest of analysis.

Our next analysis is the effect of microcell density on the energy efficiency and spectral efficiency. In Figure 9(a), 

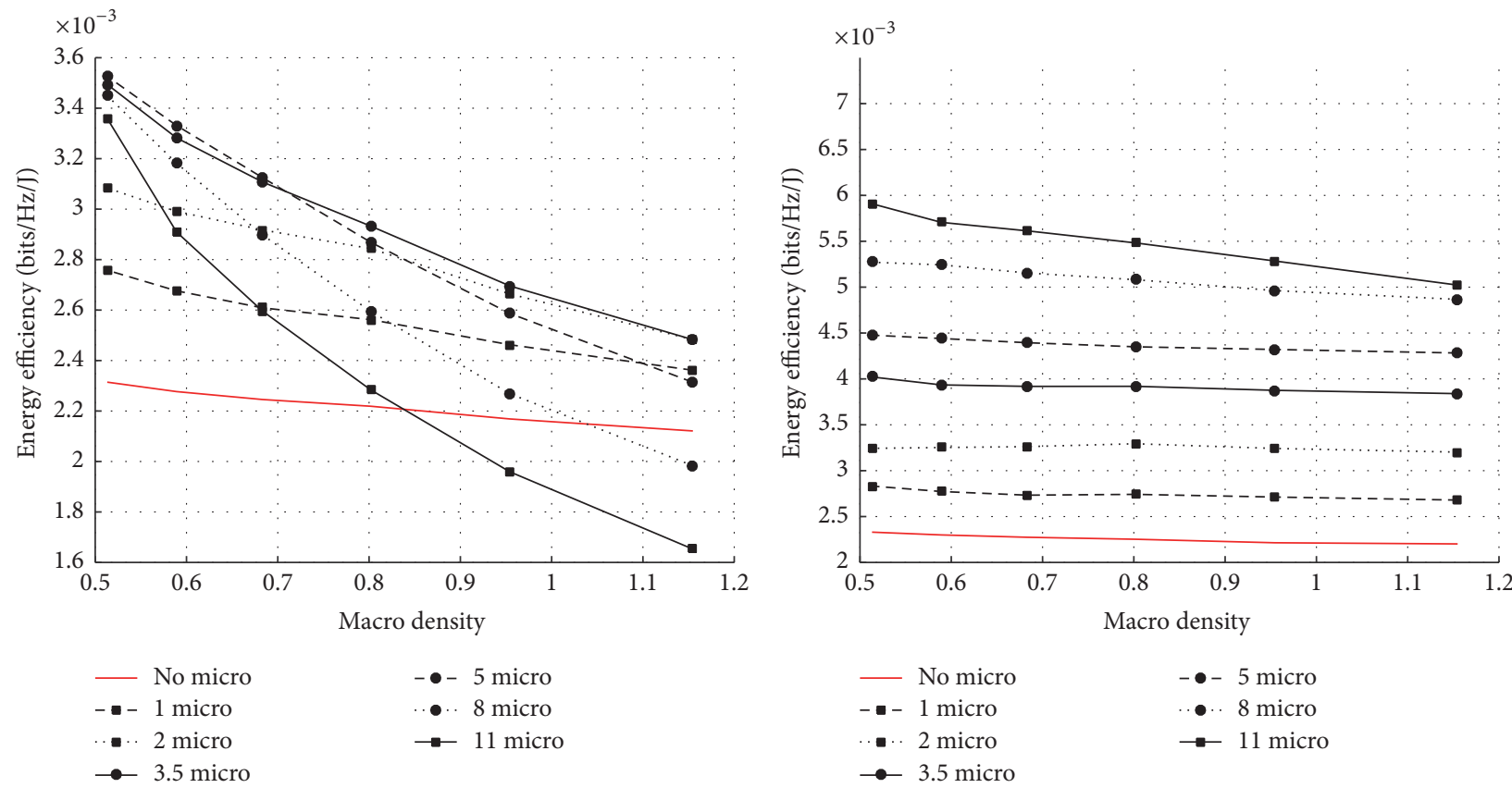

- 5 micro

- No micro
-- 1 micro
.-. 2 micro

- 5 micro

-. 8 micro

$\rightarrow 3.5$ micro

(a) 5 users $/ \mathrm{km}^{2}$

(b) 100 users $/ \mathrm{km}^{2}$

Figure 7: Energy efficiency for PPP based model.



(a) 5 users $/ \mathrm{km}^{2}$

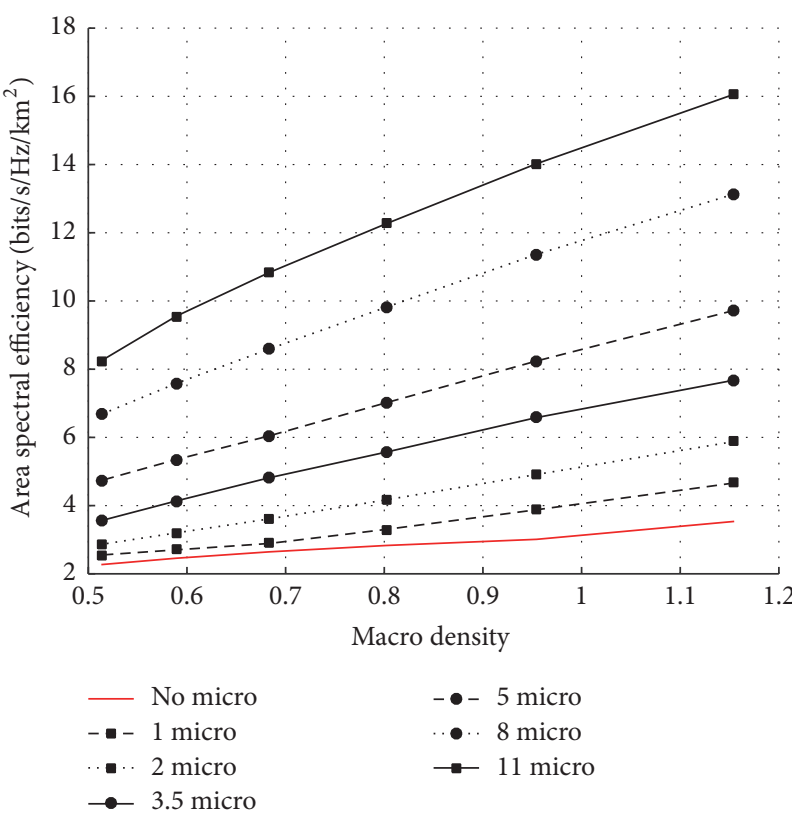

(b) 100 users $/ \mathrm{km}^{2}$

FIGURE 8: Area spectral efficiency for PPP based model.

we observe that microcell addition improves the energy efficiency for both cases. For sparse user case, 3 microcells/macrocell scenario seems to be the most energy efficient scenario whereas 11 microcells/site $\left(\lambda_{\mu}=12.7017\right)$ scenario maximizes the energy efficiency for dense user case (see Section 2 for $\lambda_{\mu}$ calculation). Furthermore, we observe in Figure 9(b) that microcell deployment improves the spectral efficiency for both cases. Note that 8 microcells/site scenario maximized the spectral efficiency when we employed the hexagonal grid model (see Figure 4(b)). However, 11 microcells/site $\left(\lambda_{\mu}=12.7017\right)$ scenario is the most spectral efficient scenario in Figure 9(b). It is necessary to note that this is not a contradiction. In the stochastic geometry model, microcell coverage regions do not necessarily overlay even 




(a) Energy efficiency

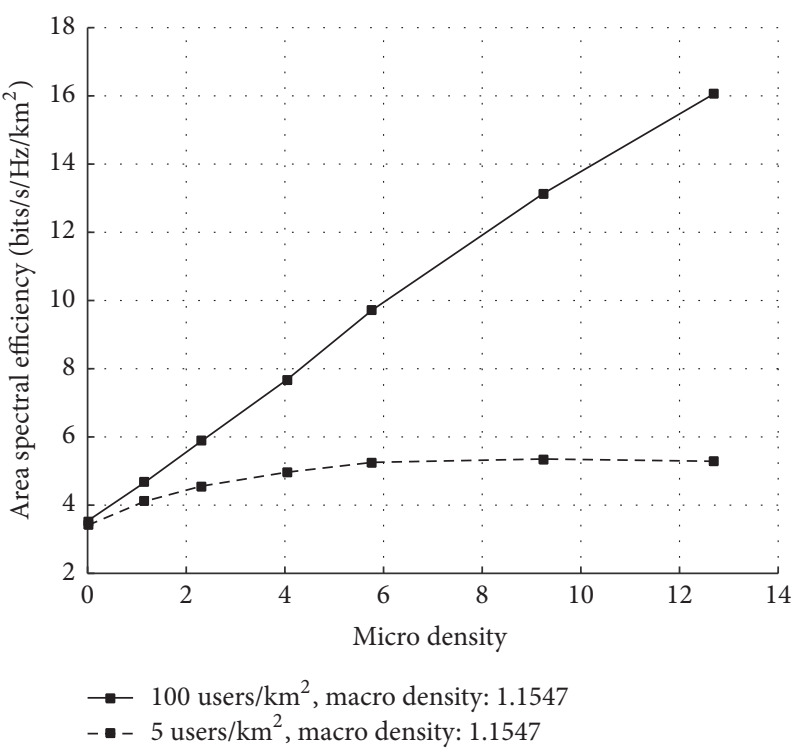

(b) Area spectral efficiency

FIgURE 9: The effect of the microcell density when $\lambda_{M}=1.1547$.

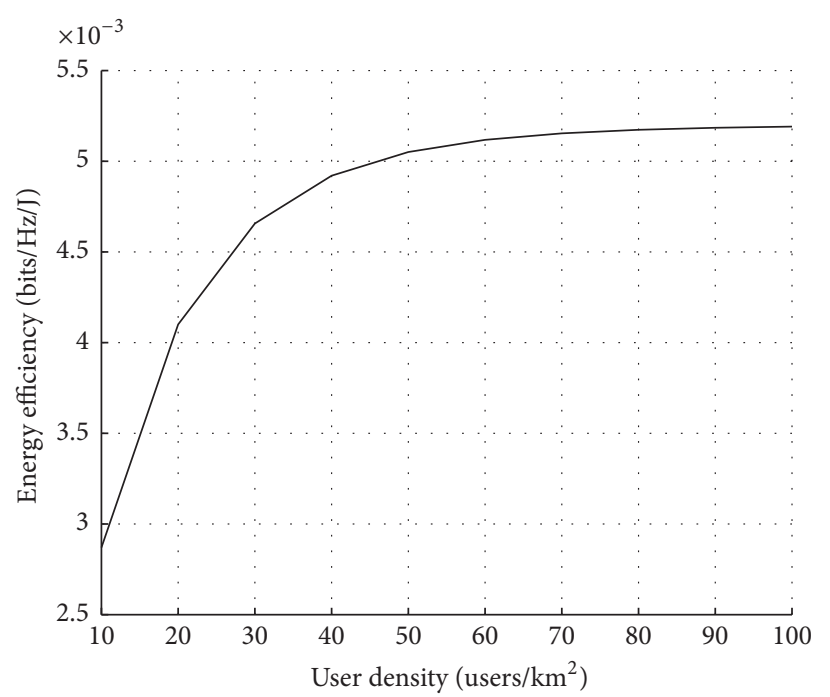

_ Macro density: 1.1547 , micro density: 12.7017

(a) Energy efficiency



_ Macro density: 1.1547 , micro density: 12.7017

(b) Area spectral efficiency

FIgURE 10: The effect of user density when $\lambda_{M}=1.1547$ and $\lambda_{\mu}=12.7017$.

if we deploy more than 8 microcells per site. Thus, spectral efficiency is expected to improve with the increasing number of microcells when we use a stochastic model.

Finally, we fix the macrocell and microcell densities at the most spectral efficient combination $\left(\lambda_{M}=1.1547\right.$ and $\left.\lambda_{\mu}=12.7017\right)$ and consider the effect of user density. As it is seen in Figure 10, increasing user density reasonably improves both energy efficiency and spectral efficiency. It is a quite intuitive result since the microcell utilization rate increases with increasing user density.

\section{Conclusion}

In this work, we investigated the effect of nonoverlay heterogeneous network planning on the energy efficiency and spectral efficiency of cellular networks. We consider two different heterogeneous deployment models, namely, the hexagonal grid model and the stochastic geometry model. We conclude that the energy efficiency depends on the macrocell density, microcell density, and user traffic. In a stochastic geometry model that better represents the real deployment 
scenarios, adding microcells is energy efficient for even sparse user scenarios. For more structured scenarios, the positive effects of adding microcells on the energy efficiency can only be seen for dense user distributions. Our future work is to approach this problem mathematically and propose an algorithm that finds the optimum energy efficiency by solving the optimum macrocell and microcell density values for a given user density. In addition, we will consider more detailed power consumption models in our future studies.

\section{Competing Interests}

The authors declare that there is no conflict of interests regarding the publication of this paper.

\section{Acknowledgments}

This work was supported by Tübitak Teydeb, Grant 15055150007, and Ericsson. The authors would like to thank Mr. Çağatay Sağında, Mr. Haydar Şahin, Mr. Faruk Bozkurt, and Mr. Turgut Erkul from Ericsson for their time and advice in the preparation of this work.

\section{References}

[1] Z. Hasan, H. Boostanimehr, and V. K. Bhargava, "Green cellular networks: a survey, some research issues and challenges," IEEE Communications Surveys and Tutorials, vol. 13, no. 4, pp. 524540, 2011.

[2] L. M. Correia, D. Zeller, O. Blume et al., "Challenges and enabling technologies for energy aware mobile radio networks," IEEE Communications Magazine, vol. 48, no. 11, pp. 66-72, 2010.

[3] C. Han, T. Harrold, S. Armour et al., "Green radio: radio techniques to enable energy-efficient wireless networks," IEEE Communications Magazine, vol. 49, no. 6, pp. 46-54, 2011.

[4] D. Feng, C. Jiang, G. Lim, L. J. Cimini, G. Feng, and G. Y. Li, "A survey of energy-efficient wireless communications," IEEE Communications Surveys and Tutorials, vol. 15, no. 1, pp. 167178, 2012.

[5] A. Damnjanovic, J. Montojo, Y. Wei et al., "A survey on 3GPP heterogeneous networks," IEEE Wireless Communications, vol. 18 , no. 3, pp. 10-21, 2011.

[6] S. Tombaz, K. W. Sung, and J. Zander, "On metrics and models for energy-efficient design of wireless access networks," IEEE Wireless Communications Letters, vol. 3, no. 6, pp. 649-652, 2014.

[7] F. Richter, A. J. Fehske, and G. P. Fettweis, "Energy efficiency aspects of base station deployment strategies for cellular networks," in Proceedings of the IEEE 70th Vehicular Technology Conference Fall (VTC '09 Fall), September 2009.

[8] A. J. Fehske, F. Richter, and G. P. Fettweis, "Energy efficiency improvements through micro sites in cellular mobile radio networks," in Proceedings of the IEEE Globecom Workshops (Gc Workshops '09), Honolulu, Hawaii, USA, November-December 2009.

[9] S. Morosi, A. Fanfani, and E. Del Re, "Network deployment and RRM strategies for green mobile communications," in Proceedings of the 18th European Wireless Conference (European Wireless), April 2012.
[10] H. Ren, M. Zhao, W. Zhou, P. Dong, and J. Kong, "Traffic-aware micro base station planning in wireless cellular networks," in Proceedings of the IEEE 78th Vehicular Technology Conference (VTC '13), IEEE, Vegas, Nev, USA, September 2013.

[11] M. Demirtaş and A. Soysal, "Energy and spectral efficient microcell deployment in heterogeneous cellular networks," in Proceedings of the IEEE 81st Vehicular Technology Conference (VTC Spring '15), Glasgow, UK, May 2015.

[12] M. Demirtas and A. Soysal, "Energy efficient microcell deployment for HetNets," in Proceedings of the 23rd Signal Processing and Communications Applications Conference (SIU '15), pp. 531534, Malatya, Turkey, May 2015.

[13] C. Xiong, G. Y. Li, S. Zhang, Y. Chen, and S. Xu, "Energy- and spectral-efficiency tradeoff in downlink OFDMA networks," IEEE Transactions on Wireless Communications, vol. 10, no. 11, pp. 3874-3886, 2011.

[14] S. Tombaz, K. W. Sung, and J. Zander, "Impact of densification on energy efficiency in wireless access networks," in Proceedings of the IEEE Globecom Workshops (GC Wkshps '12), pp. 57-62, Anaheim, Calif, USA, December 2012.

[15] J. Tang, D. K. C. So, E. Alsusa, and K. A. Hamdi, "Resource efficiency: a new paradigm on energy efficiency and spectral efficiency tradeoff," IEEE Transactions on Wireless Communications, vol. 13, no. 8, pp. 4656-4669, 2014.

[16] J. Tang, D. K. C. So, E. Alsusa, K. A. Hamdi, and A. Shojaeifard, "Resource allocation for energy efficiency optimization in heterogeneous networks," IEEE Journal on Selected Areas in Communications, vol. 33, no. 10, pp. 2104-2117, 2015.

[17] J. Tang, D. K. C. So, E. Alsusa, K. A. Hamdi, and A. Shojaeifard, "On the energy efficiency-spectral efficiency tradeoff in MIMOOFDMA broadcast channels," IEEE Transactions on Vehicular Technology, vol. 65, no. 7, pp. 5185-5199, 2016.

[18] M. Demirta and A. Soysal, "Energy and spectral efficient microcell deployment in hetero geneous cellular networks," in Proceedings of the 24th Signal Processing and Communication Application Conference (SIU '16), May 2016.

[19] J. G. Andrews, F. Baccelli, and R. K. Ganti, "A tractable approach to coverage and rate in cellular networks," IEEE Transactions on Communications, vol. 59, no. 11, pp. 3122-3134, 2011.

[20] H. S. Dhillon, R. K. Ganti, F. Baccelli, and J. G. Andrews, "Modeling and analysis of K-tier downlink heterogeneous cellular networks," IEEE Journal on Selected Areas in Communications, vol. 30, no. 3, pp. 550-560, 2012.

[21] X. Zhang, Z. Su, Z. Yan, and W. Wang, "Energy-efficiency study for two-tier heterogeneous networks (HetNet) under coverage performance constraints," Mobile Networks and Applications, vol. 18, no. 4, pp. 567-577, 2013.

[22] J. Peng, P. Hong, and K. Xue, "Energy-aware cellular deployment strategy under coverage performance constraints," IEEE Transactions on Wireless Communications, vol. 14, no. 1, pp. 6980, 2015.

[23] G. Auer, V. Giannini, C. Desset et al., "How much energy is needed to run a wireless network?" IEEE Wireless Communications, vol. 18, no. 5, pp. 40-49, 2011.

[24] 3GPP, “Tr36.814 further advancements for E-UTRA: Physical layer aspects (release-9)," Tech. Rep., 3rd Generation Partnership Project, 2009.

[25] M. S. Alouini and A. Goldsmith, "Area spectral efficiency of cellular mobile radio systems," in Proceedings of the IEEE 47th Vehicular Technology Conference, May 1997. 




\section{Enfincering}




The Scientific World Journal



\section{Hindawi}

Submit your manuscripts at

https://www.hindawi.com



International Journal of

Distributed

Sensor Networks

$-$

Joumal of

Control Science

and Engineering
